\title{
Measurement and Management of Increased Intracranial Pressure
}

\author{
Ali Sadoughi ${ }^{1}$, Igor Rybinnik ${ }^{2}$ and Rubin Cohen ${ }^{*}, 1$ \\ ${ }^{I}$ Department of Medicine, Division of Pulmonary Critical Care, Hofstra-North Shore LIJ School of Medicine, USA \\ ${ }^{2}$ Department of Neurology, Hofstra-North Shore LIJ School of Medicine, USA
}

\begin{abstract}
Increased intracranial pressure (ICP) is a serious complication of a variety of neurologic injuries and is a major challenge in intensive care units. The most common causes of increased ICP are: traumatic brain injury (TBI), stroke, neoplasms, hydrocephalus, hepatic encephalopathy, CNS venous return impairment, encephalitis, and abscesses. Prompt diagnosis and intensive monitoring and therapy of this condition are essential for successful management of this potentially devastating condition. Recent technical innovations in neuromonitoring may allow for improvement in morbidity and mortality rates attributable to elevated ICP. Normal ICP ranges from 3-15 mmHg. In routine intensive care unit (ICU) practice, the goal of ICP management is to maintain levels below $20 \mathrm{mmHg}$. Noninvasive and metabolic monitoring of ICP including imaging-clinical examination has been studied and suggested to be as efficient as the care based on invasive ICP monitoring; however its application in clinical practice is to be established. Raised intracranial pressure correlates with decreased survival and is often the only remediable element of brain pathology. While elimination of the cause of elevated ICP remains the definitive approach, there are maneuvers that should be used to decrease ICP urgently. Surgical decompression of mass effect may rapidly improve ICP elevation. Osmolar therapy, maintenance of euvolemia, cerebral metabolic suppression, and temperature control are part of the advanced management of elevated ICP.
\end{abstract}

Keywords: Intracranial pressure, neurologic intensive care, neuromonitoring, hyperosmolar therapy, transcranial doppler.

\section{INTRODUCTION}

Increased intracranial pressure (ICP) is a serious final common pathway of a variety of neurologic injuries. It is characterized by increased volume of the intracranial vault, and is a major challenge in intensive care units. Elevated intracranial pressure has consistently been associated with a poor outcome. In a review of studies of traumatic brain injury, the rate of death was $18.4 \%$ for patients with an intracranial pressure of less than $20 \mathrm{~mm} \mathrm{Hg}$ but $55.6 \%$ for those with an intracranial pressure of more than $40 \mathrm{~mm} \mathrm{Hg}$ [1].

The most common causes of increased intracranial pressure (ICP) include: traumatic brain injury (TBI), stroke, neoplasms, hydrocephalus, hepatic encephalopathy, CNS venous return impairment, encephalitis, and abscesses. (Table 1) In all the above-mentioned types of acute cerebral lesions, raised intracranial pressure has a proximate relationship to survival and is often the only remediable element of the disease [2]. The prevention of secondary brain damage from raised intracranial pressure is therefore a central focus of neurologic intensive care. Prompt diagnosis and intensive monitoring and therapy of this condition are essential for successful management of this devastating condition. Sustained elevated ICP causes brain damage and can be rapidly fatal. Recent technical innovations in

*Address correspondence to this author at the Division of Pulmonary, Sleep and Critical Care Medicine, The Hofstra North Shore-LIJ School of Medicine, 410 Lakeville Rd, Suite 107, New Hyde Park, NY 11040, USA; Tel: 01516465 5400; Fax: 01516 465- 5454; E-mail: rocohen@nshs.edu neuromonitoring may allow for improvement in morbidity and mortality rates attributable to elevated intracranial pressure (ICP). Understanding of the mechanism contributing to ICP elevation is important in order to effectively manage and reverse the underlying process before irreversible neuronal injury occurs.

\section{PATHOPHYSIOLOGY}

According to the Monroe-Kellie doctrine the cranial vault is a fixed space of about 1400 to $1700 \mathrm{ml}$ in average-sized adults. It contains three compartments: blood (10 percent $\sim 150 \mathrm{ml}$ ), cerebrospinal fluid (CSF) (10 percent $\sim 150 \mathrm{ml}$ ), and brain tissue ( 80 percent $\sim 1400 \mathrm{ml}$ ). CSF is produced by the choroid plexus in the ventricles and elsewhere in the central nervous system (CNS) at a rate of approximately 20 $\mathrm{ml} / \mathrm{hr}(500 \mathrm{ml} /$ day $)$, and drains into the venous system via the arachnoid villi and granulations. Normal ICP ranges from 50 to $200 \mathrm{mmH} 2 \mathrm{O}$ or $3-15 \mathrm{mmHg}$. In routine intensive care unit (ICU) practice, the goal of ICP management is to maintain levels below $20 \mathrm{mmHg}$ [3].

In order to maintain a constant ICP, any increase in the volume of an intracranial element must be equally compensated by a decrease in the volume of another component, otherwise ICP will increase. Initially, a small volume expansion causes only a slight elevation in ICP. CSF is displaced through the foramen magnum into the paraspinal space, blood is displaced from the intracranial to the extracranial venous system, and the brain parenchyma is compressed. However, the compliance curve is nonlinear; when these mechanisms are exhausted intracranial compliance $(\Delta$ Volume/ $\Delta$ Pressure $)$ falls sharply, and even small increases in intracranial volume can lead to dramatic 
elevations in ICP $[4,5]$. The pressure-volume relationship between ICP, volume of CSF, blood, and brain tissue, and cerebral perfusion pressure (CPP) is known as the MonroKellie doctrine or the Monro-Kellie hypothesis. As ICP reaches 50 to $60 \mathrm{~mm} \mathrm{Hg}$, it approaches arterial pressure in the vessels of the circle of Willis and brings about global brain ischemia [6].

\section{Table 1. Causes of Increased Intracranial Pressure}

\begin{tabular}{|c|}
\hline Intracranial mass lesions \\
\hline Hemorrhage (Epidural, Subdural, intraparenchymal) \\
\hline Brain tumor \\
\hline Cerebral abscess \\
\hline Increased brain volume (cytotoxic edema and/or osmotic edema) \\
\hline Ischemic stroke \\
\hline Global hypoxia \\
\hline Reye's syndrome \\
\hline Acute hyponatremia \\
\hline Hepatic encephalopathy \\
\hline Idiopathic (pseudotomor cerebri) \\
\hline $\begin{array}{l}\text { Toxins and medications (lead, tetracycline, doxycycline, rofecoxib, } \\
\text { retinoic acid) }\end{array}$ \\
\hline Increased CSF volume \\
\hline Hydrocephalus (Communicating, Non-communicating) \\
\hline Choroid plexus papilloma \\
\hline Decreased CSF absorption (ie, venous sinus thrombosis) \\
\hline CSF outflow obstruction from leptomeningeal metastasis \\
\hline $\begin{array}{l}\text { Increased blood volume (vasogenic edema, breakdown of tight } \\
\text { endothelial junctions which make up the blood-brain barrier (BBB)) }\end{array}$ \\
\hline Impaired auto-regulation (ie, endarterectomy) \\
\hline Traumatic brain injury \\
\hline Tumoral associated vasogenic edema \\
\hline Meningitis \\
\hline Encephalitis \\
\hline Vasculitis \\
\hline Hypertensive encephalopathy \\
\hline Eclampsia \\
\hline Subarachnoid hemorrhage \\
\hline Dural sinus thrombosis \\
\hline Altitude-related cerebral edema (HACE) \\
\hline Hypoxia \\
\hline Hypercarbia \\
\hline Hyperpyrexia \\
\hline Seizure \\
\hline Jugular venous obstruction \\
\hline $\begin{array}{l}\text { Mechanical ventilation (when peak and expiratory pressure }>\text { baseline } \\
\text { intracranial pressure) }\end{array}$ \\
\hline
\end{tabular}

\section{CEREBRAL BLOOD FLOW AND PERFUSION PRESSURE}

Systemic mean arterial pressure (MAP) is a main factor in maintaining cerebral perfusion. Cerebral perfusion pressure (CPP), defined as the mean arterial pressure (MAP) minus ICP (CPP = MAP- ICP) plays an important role in ICP management. Normally cerebral blood flow (CBF) is about $50 \mathrm{ml} / 100 \mathrm{~g}$ per minute and equals to CPP divided by cerebral vascular resistance $(\mathrm{CVR})(\mathrm{CBF}=\mathrm{CPP} / \mathrm{CVR})$. The brain's blood vessels are able to vasoconstrict or vasodilate in response to a number of stimuli. This autoregulation maintains $\mathrm{CBF}$ at a constant level over a wide range of CPPs (from 50 to $150 \mathrm{mmHg}$ ). When $\mathrm{CBF}$ falls below $12 \mathrm{~mL} / 100$ $\mathrm{g}$ per minute, irreversible ischemic injury occurs [7]. If CPP levels fall below the lower limit of autoregulation, $\mathrm{CBF}$ will fall and contribute to oligemia. If CPP exceeds the upper limit of autoregulation, an excess of CBF would occur beyond what is necessary for normal brain metabolism. In different brain pathologies such as stroke or trauma, the brain's autoregulation is impaired and the CBF becomes more sensitive to minor changes in CPP. The autoregulatory curve is shifted to the left in children and shifted to the right in patients with chronic hypertension. Optimally, CPP should be kept above $70 \mathrm{mmHg}$ to avoid ischemia and below $120 \mathrm{mmHg}$ to avoid hyperperfusion [8]. Hypercarbia and hypoxemia leads to vasodilation and decreased $\mathrm{CBF}$, although cerebral vessels are less responsive to changes in $\mathrm{PaO} 2$ than rapid changes in $\mathrm{PaCO} 2$.

\section{SIGNS AND SYMPTOMS}

Symptoms and signs of increased ICP are nonspecific but in the right clinical setting may lead to prompt diagnostic and treatment approaches. Headache, vomiting, disorientation, and lethargy are the main symptoms. Signs include hypertension, depressed global consciousness, papilledema, cranial nerve VI palsy, spontaneous periorbital bruising, projectile vomiting and Cushing's triad (hypertension, bradycardia, and irregular respiration). (Table 2) Cushing's triad may be seen in brain stem herniation without significant elevation of ICP. Some of the symptoms and signs are caused by cerebral herniation and not necessarily by elevated ICP, including cranial nerve III palsy, motor posturing, lower extremity rigidity, loss of lateral ocular movements, and aberrations of respiration. Brain tissue displacement and herniation occur when compartmentalized mass effect leads to ICP gradients i.e. regional ICP differences. Two major consequences of elevated ICP are hypoxic-ischemic injury resulting from reduction of $\mathrm{CPP}$ and $\mathrm{CBF}$, and mechanical compression and herniation of brain tissue leading to brain damage or death.

Table 2. Clinical Manifestation of Increased ICP

Symptoms:
Headache
Vomiting
Disorientation
Lethargy
Signs:
Depressed level of consciousness (lethargy, stupor, coma)
Hypertension, with or without bradycardia
Papilledema
Sixth cranial nerve palsy
Cushing's triad (hypertension, bradycardia, and irregular respiration)
Spontaneous periorbital bruising

Because of the poor correlation between clinical signs and ICP, the only way to properly diagnose increased ICP is to directly or indirectly measure it. Use of radiologic studies including CT scans may support diagnosis; however elevated 
ICP may occur without radiologic findings on initial CT scan [9].

\section{ICP MONITORING}

Empiric treatment of suspected elevated ICP is often necessary especially in urgent case scenarios; however without measurement of ICP, most of the therapies are not satisfactory, especially because they are directed at optimizing the CPP, which cannot be calculated without knowing the ICP. Invasive monitoring of ICP may be indicated in patients who meet all three of the following criteria [4]:

1. The patient is suspected to be at risk for elevated ICP.

2. The patient is comatose (Glasgow coma scale score $\leq$ 8).

3. The prognosis is such that aggressive ICU treatment is indicated.

Suspicion of elevated ICP generally is based on clinical findings and the results of a computed tomography (CT) scan and the patient's medical history. Although CT scans may show significant intracranial mass effect with midline shift or effacement of the basal cisterns, patients with initial normal CT scans may have elevated ICP. In a prospective study of patients with head injury, elevated ICP occured in approximately $10-15 \%$ of patients with initial normal CT scans [9]. This risk is even higher in patients more than 40 years old, with motor posturing, or with hypotension (systolic blood pressure $<90 \mathrm{mmHg}$ ) [10]. Guidelines for the use of ICP monitoring were established for traumatic brain injury, and for increased ICP associated with conditions other than trauma the guidelines are less clear. However, clinical deterioration and imaging consistent with mass effect may serve as important selection criteria in patients with non-traumatic intracranial hypertension. In a controlled trial of 324 patients with severe traumatic brain injury the care focused on maintaining monitored intracranial pressure at $20 \mathrm{~mm} \mathrm{Hg}$ or less with intraparenchymal ICP monitoring was compared to care based on imaging and clinical examination [11]. While this study was somewhat underpowered to draw a definite conclusion and did not challenge the importance of ICP monitoring it showed that imaging-clinical examination protocol might provide an appropriate control of intracranial pressure.

\section{INVASIVE ICP MONITORING DEVICES}

It should be noted that most treatments for elevated ICP will lose their efficacy with prolonged use, and should be withheld as soon as ICP is corrected, making a further case for close ICP monitoring. There are four main anatomical sites used in the clinical measurement of ICP: intraventricular, intraparenchymal, subarachnoid, and epidural [12]. Each technique requires a unique monitoring system, and has associated advantages and disadvantages.

\section{INTRAVENTRICULAR CATHETERS}

These devices are considered the gold standard of ICP monitoring, and directly connect the intracranial space to an external pressure transducer. The catheter is usually connected to both a pressure transducer and an external drainage system via a three-way stopcock. The chief advantage of such a catheter is the ability to both measure and lower ICP by CSF drainage and allows in situ calibration. The system can then be set for continuous ICP monitoring with intermittent CSF drainage or continuous drainage with intermittent ICP measurement. The main disadvantage is the high risk of infection (ventriculitis or meningitis) occurring in $10-20 \%$ of patients and increases dramatically after 5 days [13]. The other disadvantages of this type of monitoring device include blockage, increased risk of hemorrhage, necessity to readjust the transducer position with the level of the patient's head (zero should be set at the external auditory meatus), and technically difficult placement into small ventricles when there is cerebral edema or ventricular compression with resulting erroneously low ICP values.

\section{INTRAPARENCHYMAL PRESSURE TRANSDUCERS}

These are equipped with either a fiberoptic or electronic pressure transducer at their tip, and are inserted into the brain parenchyma via a small burr hole drilled in the skull. They are easier to place and have lower risk of infection and hemorrhage compared to intraventricular catheters [14-16]. These devices only need to be calibrated once prior to insertion, and the accuracy of ICP measurements is generally superior to those provided by subarachnoid bolts or epidural transducers, however there are reports of decreasing accuracy over several days.

\section{SUBARACHNOID BOLTS}

This is also a fluid-coupled system connecting the intracranial space to an external transducer via saline-filled tubing. The subarachnoid bolt is actually a hollow screw that is inserted via a burr hole. The dura at the base of the bolt is perforated, allowing the subarachnoid CSF to fill the bolt and connect to the transducer. Although the infection risk is low, these devices are prone to error, including underestimation of ICP, screw displacement, and occlusion by debris [12].

\section{EPIDURAL TRANSDUCERS}

These devices are inserted deep into the inner table of the skull and rest against the dura. They have a lower infection rate, but are prone to malfunction, displacement, and baseline drift after more than a few days of use. Much of the inaccuracy results from having the relatively inelastic dura between the sensor tip and the subarachnoid space, thus have limited clinical utility. They are used in the management of patients with coagulopathy such as those with hepatic encephalopathy $[17,18]$.

\section{ICP WAVEFORMS}

Normal ICP waveforms are similar to the arterial waveform, with a first peak (percussion wave) correlating with systole, a second peak (dicrotic wave) correlating with aortic valve closure, and a third peak (tidal wave) correlating with antegrade arterial flow during diastole; as intracranial compliance falls, the morphology of the ICP waveform also changes, in that the amplitude of the dicrotic wave, the second peak, initially equals and then exceeds the amplitude of the percussion wave [19] (Fig. 1). 


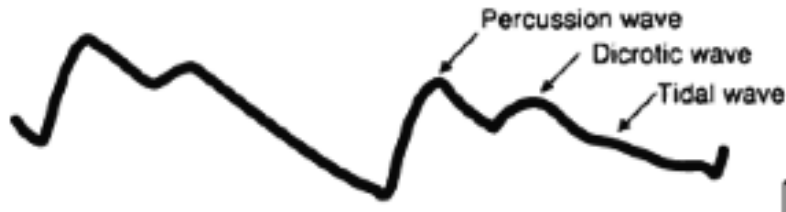

Normal ICP; normal compliance

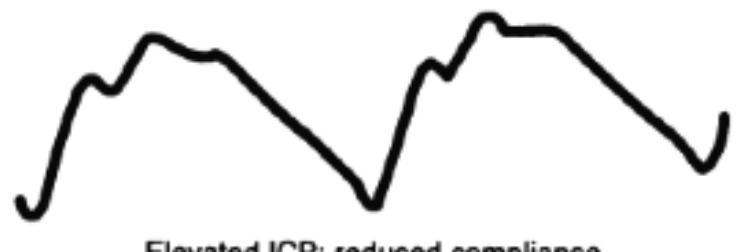

Elevated ICP; reduced compliance

\section{$1 \mathrm{sec}$}

Fig. (1). ICP waveform in conditions of normal (top) and abnormal (bottom) intracranial compliance. (Form Chestnut RM, Marshall LF. Treatment of abnormal intracranial pressure. Neurosurg Clin N Am 1991; 2: 267-284).

The relative state of intracranial compliance can be assessed by inspection of the ICP waveform. Normally ICP increases slightly $(2-3 \mathrm{mmHg})$ with each arterial pulse because of transient increases in cerebral blood volume (CBV). However, with increased ICP, intracranial pulse pressure also increases $(10-15 \mathrm{mmHg})$. Pathological A waves (also called plateau waves) are abrupt, marked elevations in ICP of 50 to $100 \mathrm{mmHg}$, which usually last for minutes to hours. The presence of A waves signifies a loss of intracranial compliance, and heralds imminent decompensation of autoregulatory mechanisms, suggestive of urgent need for intervention to decrease ICP [20, 21].

\section{NONINVASIVE ICP MONITORING}

Noninvasive and metabolic monitoring of ICP has been studied and recently been validated in a clinical trial, however its application in clinical practice is not yet established. Based on a comprehensive review article [22], several methods have been employed to estimate intracranial pressure, including computed tomography, magnetic resonance imaging, transcranial Doppler sonography, nearinfrared spectroscopy, and visual-evoked potentials. In addition, multiple techniques of measuring the optic nerve and the optic nerve sheath diameter have been studied. Noninvasive measurement of intracranial pressure can be invaluable in the management of critically ill patients.

\section{TRANSCRANIAL DOPPLER (TCD) ULTRA- SONOGRAPHY}

Transcranial Doppler (TCD) ultrasonography, which measures the velocity of blood flow in the basal cerebral arteries, shows characteristic changes with increasing ICP. TCD can be used to estimate ICP based on characteristic changes in waveforms that occur in response to increased resistance to cerebral blood flow [23]. It allows the estimation of CPP through pulsatility index (PI) [24] [PI = peak systolic velocity - end diastolic velocity / mean flow velocity]. It has emerged as a surrogate marker for ICP, especially in cases, such as those of severe coagulopathy with hepatic failure, where insertion of invasive ICP monitoring devices carries increased risk. TCD PI is a helpful tool to guide the use of hyperosmolar therapy in various conditions with intracranial hypertension [25]. As CPP falls, diastolic velocity decreases and pulsatility increases, reflecting increased distal vascular resistance to flow [26, 27]. In intracerebral hemorrhage with space occupying lesion, lateralized asymmetries in TCD pulsatility index correlate with compartmentalized ICP gradients [28].

A prospective study of eighty-one patients with various intracranial disorders (subarachnoid hemorrhage, closed head injury, other neurosurgical disorders) who had an intraventricular catheter for registration of the ICP were investigated: A total of 658 TCD measurements were made with ICP registrations in parallel with all TCD measurements. Independent of intracranial pathology, a significantly strong positive correlation between PI and intraventricular ICP monitoring was found (correlation coefficient of $0.938, p<0.0001$ ). Notably, the correlation was still strong in the patient subgroup demonstrating mean flow volume $(\mathrm{mFV})$ values above and below normal interval. In the ICP interval between 5 to $40 \mathrm{~mm} \mathrm{Hg}$ the correlation between ICP and PI enabled an estimation of ICP from the PI values with an SD of $2.5(\mathrm{ICP}=10.93 \times \mathrm{PI}-1.28)$ [29]. In a prospective study of forty-eight patients with an acute spontaneous supratentorial intracranial hemorrhage, the PI from the unaffected hemisphere was correlated with mortality (OR 2.3, CI 0.92 to 5.72, $\mathrm{p}=0.07$ ). A cutoff for PI from the unaffected hemisphere of 1.75 showed a specificity of $94 \%$ and a sensitivity of $80 \%$ as a predictor of death at 30 days [30]. Promising new applications using ultrasound technology to estimate ICP noninvasively have been described, but need to be validated in large clinical setting [31]. TCD sensitivity for vasospasm varies between 50 and $100 \%[32,33]$ and is vessel-dependent due to location and size, but has a specificity of $>90 \%$ as compared to the gold standard of digital subtraction angiography. TCD is only a single snapshot in time rather than continuous monitoring of ICP and is also heavily operator-dependent in terms of education and experience.

\section{OPTIC NERVE SHEATH DIAMETER}

The other promising technique for noninvasive ICP monitoring is the optic nerve sheath diameter measurement (ONSD) by ultrasound, which is shown to be an accurate diagnostic tool for detection of intracranial hypertension. A number of studies have found that diameters of 5 to $6 \mathrm{~mm}$ have the ability to discriminate between normal and elevated ICP in patients with intracranial hemorrhage and traumatic brain injury $[34,35]$. Limitation to its use are patients with chronic ocular disease and malignant hypertension. Interpretation of ultrasonography of ONSD should be combined with a set of clinical and radiological signs. This technique is reproducible with median intra-observer reliability of $0.2 \mathrm{~mm}(0.1-0.5 \mathrm{~mm})$ and median interobserver reliability varying from 0.2 to $0.3 \mathrm{~mm}$. Ultrasonography of ONSD may allow for earlier management of elevated ICP when invasive ICP monitoring is not available or before its placement. 
MONITORING OF MIDLINE SHIFT BY TRANSCRANIAL DUPLEX SONOGRAPHY

Midline shift (MLS) is a known prognostic factor for unfavorable outcome after intracranial hemorrhage (ICH). Transcranial duplex sonography (TDS) is a useful noninvasive bedside alternative to $\mathrm{CT}$ scan, avoiding increased radiation exposure and repeated transportation of critically ill patients, to monitor MLS in early patient management after ICH. TDS monitoring of the early dynamics of MLS reliably predicts early mortality and outcome in conservatively treated hemispheric ischemic stroke [36]. Studies revealed a high correlation of the imaging of the third ventricle between TDS and CT and a robust inter- and intra-observer reliability. In addition to the monitoring of MLS, serial TDS examinations allow the assessment of lateral ventricular width, ventricular involvement of the ICH, monitoring of post-hemorrhagic hydrocephalus and detection of early hematoma growth. A cut-off MLS increase of $5 \mathrm{~mm}$ over $24 \mathrm{~h}$ identifies patients with re-bleeding with a sensitivity of $100 \%$, although its specificity and positive predictive value remain low [26]. However, TDS is unlikely to replace CT scanning in well resourced healthcare systems.

\section{ELECTROENCEPHALOGRAPHY AND SOMATO- SENSORY EVOKED POTENTIALS MONITORING}

Electroencephalography (EEG) in the ICU is being increasingly recognized as a means to monitor cerebral function and metabolism. Continuous EEG monitoring (cEEG) is most commonly used to detect and guide treatment of nonconvulsive seizures, including after convulsive status epilepticus. In addition, cEEG is used to guide management of pharmacological coma for treatment of increased intracranial pressure. An emerging application for cEEG is to detect new or worsening brain ischemia in patients at high risk, to provide continuous information about changes in brain function in real time at the bedside and to alert clinicians to any acute brain event, including seizures, ischemia, increasing intracranial pressure, hemorrhage, and even systemic abnormalities affecting the brain, such as hypoxia, hypotension, acidosis, and others [37].

In a study of sixty-eight patients with elevated ICP patients (Traumatic brain injury (TBI), intracranial hemorrhage (ICH), and ischemic stroke; GCS < 9), the concept of detecting neurologic deterioration using EEG and somatosensory evoked potential (SEP) was defined and compared with ICP monitoring. $68 \%$ of the patients with elevated ICP had worsening of evoked responses either before or during the worsened ICP. In $25-30 \%$ of deteriorating patients SEP were the first hallmark of the worsening of brain function, preceding an ICP increase by many hours. They found that there is a range of values (20$40 \mathrm{mmHg}$ ) where ICP increase is scarcely indicative of the occurrence of clinical state deterioration, while SEP changes are able to identify the deterioration of brain function. This may explain why the role of ICP monitoring in the management of acute encephalopathy remains controversial and that neurophysiological monitoring could help interpret and manage ICP trends and their modifications. Beyond the fact that their alterations precede ICP increases, SEP should also be considered a useful complementary tool. While ICP monitoring provides pressure values from which we can extrapolate cerebral perfusion, SEP monitoring reflects the extent to which cerebral parenchyma remains metabolically active and viable during acute brain injury. It is very important to associate SEP and EEG because in deeply sedated patients only SEP allow to monitor brain damage evolution when EEG is scarcely valuable [38].

\section{TREATMENT}

The prevention of secondary brain damage from raised intracranial pressure is the central focus of neurologic intensive care. The critical nature of elevated intracranial pressure (ICP) mandates the implementation of its treatment as quickly as possible. The fundamental abnormality common to these diverse disease states is an increase in intracranial volume. Accordingly, all treatments for elevated ICP work by reducing intracranial volume. The primary goal of ICP management is to maintain ICP below $20 \mathrm{mmHg}$ and CPP above $60 \mathrm{mmHg}$. While elimination of the cause of elevated ICP remains the definitive approach, there are maneuvers that should be used to decrease ICP urgently, while CPP management should be emphasized at the same time. The stepwise protocol shown below reflects these considerations.

\section{Surgical decompression}

2. Sedation

3. CPP optimization

4. Osmotherapy

5. Hyperventilation

6. High-dose pentobarbital therapy

7. Hypothermia [4].

Existing trials have been too small to support or refute the existence of a real benefit from using hyperventilation, mannitol, CSF drainage, barbiturates, or corticosteroids [39]. Until these uncertainties are resolved, clinicians should continue to make treatment decisions based on their judgment and experience according to the best available evidence [40].

\section{INITIAL STEPS}

Optimization of oxygenation (O2 saturation $>94 \%$ or $\mathrm{Pao} 2>80 \mathrm{~mm} \mathrm{Hg}$ ) and cerebral blood flow (systolic BP greater than $90 \mathrm{~mm} \mathrm{Hg}$ ) are essential. BP should be sufficient to maintain CPP $>60 \mathrm{mmHg}$ and pressers can be used safely, especially when iatrogenic hypotension occurs with sedation [41]. The treatment of hypertension is discussed in more detail in another article in this supplement. Normalization of blood pressure in patients with chronic hypertension in whom the autoregulatory curve has shifted to the right should be avoided, unless other considerations exist, namely acute intracranial hemorrhage where BP lowering decreases hematoma expansion.

Addressing venous outflow obstruction with upright midline head positioning is a critical initial step. Head of the bed should be maintained at $30^{\circ}$, and the patient's head should remain in midline positioning, without jugular compression, to promote venous return. Head elevation in excess of 45 degrees should generally be avoided because 
paradoxical increases in ICP can occur in response to excessive CPP reduction [42]. Important maneuvers include reducing excessive flexion or rotation of the neck, avoiding restrictive neck taping, and minimizing stimuli that could induce cough and valsalva responses, such as endotracheal suctioning.

Maintenance of euvolemia and strict monitoring of fluid balance are necessary. Only isotonic fluids should be used and hypotonic fluids such as $5 \%$ dextrose or $0.45 \%$ (halfnormal) saline should be strictly avoided. Systemic hypoosmolality $(<280 \mathrm{mOsm} / \mathrm{L})$ should be aggressively reversed. Dehydration therapy is not recommended, and in fact, hypovolemia may lead to inadequate CPP and consequent increase in ICP [43]. Choice of optimal fluids for resuscitation remains uncertain, as studies comparing colloids with crystalloids have been inconclusive. However, one large study suggested that in patients with traumatic brain injury, albumin may be harmful and should be avoided, as it was associated with a higher mortality compared with normal saline [44].

Keeping patients appropriately sedated, treating agitation and controlling the pain with analgesia can decrease ICP by reducing metabolic demand, ventilator asynchrony, venous congestion, and the sympathetic responses of hypertension and tachycardia [45].

Fever increases brain metabolism and should be treated aggressively. It increases ICP by increasing cerebral metabolism and blood flow, and have been shown to exacerbate hypoxic-ischemic neuronal injury in animal models [46, 47]. A French study showed that fever control using external cooling was safe and decreased vasopressor requirements and early mortality in septic shock [48]. Therefore, aggressive treatment of fever, including acetaminophen and mechanical cooling, is apparently safe and is recommended in patients with increased ICP with sustained fevers in excess of $38.3^{\circ} \mathrm{C}$.

Seizures and even non-convulsive status epilepticus are strikingly common in the injured brain [49]. These increase cerebral metabolic demand and induce hyperemia, which can significantly contribute to the elevation of ICP [50]. Therefore, prophylactic antiepileptic medications should be considered, especially in patients with large focal cortical lesions with significant mass effect and midline shift, and there should be a low threshold to order video EEG monitoring in a comatose patient.

Dexamethasone and other steroids should not be used for treatment for ICP, except in tumor patients, because they are ineffective against cytotoxic edema. There is generally no role for steroids in the treatment of mass effect related to cerebral infarction, intracerebral hemorrhage, or TBI. In MRC CRASH trial the effect of corticosteroids on death and disability after head injury within 8 hour of injury was examined. The risk of death was higher in the corticosteroid group than in the placebo group (1248 [25.7\%] vs 1075 [22.3\%] deaths; relative risk $1.15,95 \%$ CI 1.07-1.24; $\mathrm{p}=0.0001)$, as was the risk of death or severe disability $(1828$ [38.1\%] vs 1728 [36.3\%] dead or severely disabled; 1.05 , $0.99-1.10 ; \mathrm{p}=0.079)$. They conclude that corticosteroids should not be used routinely in the treatment of head injury [51]. However, vasogenic edema related to neoplasm may be steroid responsive, and dexamethasone can lead to dramatic reductions in lesion volume. Corticosteroid use for brain abscess is controversial and is used when a significant mass effect is visible on imaging and the patient's mental status is depressed. When used to reduce cerebral edema, therapy should be of short duration [52].

\section{HYPEROSMOLAR THERAPY}

Osmotic agents reduce brain tissue volume by drawing free water out of brain tissue and into the systemic circulation, where it is then excreted by the kidneys [53, 54]. The beneficial effect of hyperosmolar therapy requires that the blood-brain barrier (BBB) be intact. In regions of braintissue damage, as in traumatic contusion, the barrier is disrupted and allows equilibration of molecules between blood and the interstitial fluid of the brain. Thus, hyperosmolar agents exert their effect largely by removing water from the remaining normal brain tissue [6]. Most of the reduction of brain volume occurs during and soon after the period of maximal osmolarity induced by the infusion of a hyperosmolar agent. The brain slowly accommodates to serum hyperosmolarity by raising intracellular solute concentrations through a number of means, most of which are not clearly understood.

Clinical evidence demonstrates the efficacy of mannitol and hypertonic saline for acute intracranial hypertension in the setting of TBI, edema secondary to tumor, ICH, SAH, and stroke [55]. Mannitol and hypertonic saline have been compared in at least five randomized trials of patients with elevated ICP from a variety of causes (traumatic brain injury, stroke, tumors) [56]. A meta-analysis of these trials found that hypertonic saline appeared to have greater efficacy in managing elevated ICP, but the effect on clinical outcomes were not assessed [57].

Mannitol dosing has been suggested between 0.18 and $2.5 \mathrm{~g} / \mathrm{kg}$, although doses $<0.5 \mathrm{~g} / \mathrm{kg}$ are less efficacious and less durable, and a positive correlation has been demonstrated between dose and magnitude of ICP reduction. Bolus dosing of hypertonic saline (in concentrations ranging from 1.5 to $23.4 \%$ ) can be generalized as ranging from 240 $\mathrm{mOsm} /$ dose (e.g., $30 \mathrm{ml}$ of $23.4 \%$ ) to $640 \mathrm{mOsm} /$ dose (e.g., $250 \mathrm{ml}$ of $7.5 \%$ ). The amount of hypertonic saline that is required to reach a target serum sodium concentration can be approximated from the following formula: sodium requirement in millimoles $=$ (lean body weight in kilograms $\times$ the proportion of weight that is water, which is 0.5 for a woman and 0.6 for a man) $\times$ (desired sodium - current sodium in millimoles per liter $\mathrm{mmol} / \mathrm{L})$. The required volume in milliliters is then calculated as the sodium requirement divided by the sodium concentration of the chosen solution. Dosing of infusion therapy of hypertonic saline has been effective using $3 \% \mathrm{NaCl}$ at $0.1-2.0 \mathrm{ml} / \mathrm{kg} / \mathrm{h}$ on a sliding scale titrating to serum sodium concentrations of 145-155mmol/L [56]. Clear guidelines and specific targets for an optimal serum sodium concentration are not well established. In our ICU we usually use 3\% hypertonic saline, started at $30 \mathrm{ml} / \mathrm{h}$ via a peripheral vein with the goal of serum sodium concentration of $145-155 \mathrm{mmol} / \mathrm{L}$ to be reached within 6 hours. The goal can be reached faster with administration of another simultaneous 3\% hypertonic saline infusion via a second peripheral IV, or alternatively a higher 
saline concentration via a central line. The serum sodium goal is usually maintained for at least 72 hours. In our practice we rarely use mannitol.

The rate of administration of an osmolar load may affect the efficacy of lowering ICP. Sustained administration and lower weight-based dosing of mannitol have been shown to have a less pronounced and less enduring impact on elevated ICP. Bolus dosing may create a higher osmolar gradient across the BBB, ultimately inducing a larger decrease in parenchymal fluid. In refractory cases, mannitol and hypertonic sodium may be alternated or given simultaneously.

Because of the concern for mannitol-induced renal failure, the osmolar gap (the difference between the calculated and measured serum osmolarity) should be kept less than $55 \mathrm{mOsm} / \mathrm{kg}$ [57]. Monitoring serum osmolarity in these patients does not appear to predict the development or risk of acute renal failure [calculated osmolality formula: $2(\mathrm{Na})+$ glucose/18 + blood urea nitrogen/2.8] [58, 59]. Withholding mannitol should also be considered if the serum osmolality exceeds $320 \mathrm{mOsm} / \mathrm{L}$, although little evidence exists to support this number as the upper limit.

Hypertonic saline infusion is capable of achieving ICP reduction for a period $<72$ hours but this effect may not be durable with prolonged therapy. Prolonged use of hypertonic saline allows the cerebral homeostatic mechanism to equilibrate the osmotic gradient and results in hypothetical rebound edema and intracranial hypertension if hypertonic saline is discontinued abruptly. Rebound ICP increase has been reported with hyperosmolar therapy (particularly with mannitol), but it is unclear whether this increase is secondary to iatrogenic reversal of the hyperosmolar gradient, or whether true spontaneous reversal takes place.

In the absence of evidence of clinical complications, and with both animal and human data suggesting the safety of peripheral hypertonic saline boluses, the practice of withholding hyperosmolar therapy for lack of central venous access appears unjustified.

In the absence of preexisting hyponatremia and predisposing comorbidities, there is little evidence to suggest that rapid elevation of serum sodium with hypertonic saline during initiation of acute hyperosmolar therapy presents a significant central pontine myelinolysis (CPM) risk for most patients. Other adverse effects include electrolyte abnormalities, acidosis, hypotension, and congestive heart failure. If hyperchloremic metabolic acidosis develops with hypertonic saline administration, hypertonic sodium bicarbonate solutions may serve as an effective alternative [60]. On the other hand, hypertonic saline has a clear advantage over mannitol in hypovolemic and hypotensive patients. Mannitol is relatively contraindicated in hypovolemic patients because of its diuretics effects; whereas hypertonic saline augments intravascular volume and may increase blood pressure in addition to decreasing ICP.

\section{HYPERVENTILATION}

Hyperventilation should be used only acutely to achieve $\mathrm{PaCO} 2$ to 26 to $30 \mathrm{mmHg}$; it rapidly reduces ICP through vasoconstriction and decreasing the intracranial blood volume. The vasoconstrictive effect on cerebral arterioles is temporary lasting less than 24 hours. As the CSF $\mathrm{pH}$ equilibrates to the new $\mathrm{PaCO}_{2}$ level, the cerebral arterioles redilate, possibly to a larger caliber than at baseline, and the initial reduction in cerebral blood volume comes at the cost of a possible rebound phase of increased ICP. If used, hyperventilation should be tapered slowly over 4-6 hours to avoid vasodilatation and rebound increases in ICP [4].

Hyperventilation results in vasoconstriction and while this could decrease ICP, concurrently, a critical decrease in local cerebral perfusion may ensue that can potentially worsen neurologic injury, particularly in the first 24 to 48 hours $[61,62]$. Although hyperventilation-induced ischemia has not been clearly shown, routine chronic hyperventilation (to $\mathrm{PaCO} 2$ of $20-25 \mathrm{~mm} \mathrm{Hg}$ ) has been demonstrated to have a detrimental effect on outcome in one randomized clinical trial [63]. Therefore, hyperventilation is used most effectively as a temporizing measure until more definitive treatments for increase intracranial pressure are instituted.

\section{BARBITURATES}

Barbiturate therapy to induce electroencephalographic burst suppression has been a mainstay of pharmacologic metabolic suppression for elevated ICP, although it is not indicated for prophylactic administration [64]. The use of barbiturates is predicated on their ability to reduce brain metabolism and cerebral blood flow, thus lowering ICP and exerting a neuroprotective effect. Pentobarbital is generally used, with a loading dose of 5 to $20 \mathrm{mg} / \mathrm{kg}$ as a bolus, followed by 1 to $4 \mathrm{mg} / \mathrm{kg}$ per hour. Significant morbidity, namely hypotension that usually requires the use of vasopressors, may be associated with this therapy, therefore it should be reserved for cases of ICP refractory to standard first-line medical care. Close monitoring of ICP and CPP is mandatory. Continuous EEG monitoring is generally used because barbiturates use is associated with loss of the neurologic examination; EEG burst suppression is an indication of maximal dosing. A randomized trial of 73 patients with elevations in ICP refractory to standard therapy showed that patients treated with pentobarbital were 50 percent more likely to have their ICP controlled, but there was no difference in clinical outcomes between groups, leaving the therapeutic value of this treatment unclear [65].

\section{THERAPEUTIC HYPOTHERMIA}

Hypothermia decreases cerebral metabolism and may reduce $\mathrm{CBF}$ and ICP. First reported as a treatment for brain injury in the $1950 \mathrm{~s}$, most evidence shows that cooling can be effective in patients with severe TBI and intracranial hypertension provided that treatment is initiated early, continued for an appropriate duration of time (2-5 days), and followed by a gradual rewarming. Hypothermia is clearly effective in controlling intracranial hypertension. However, positive effects on survival and neurological outcome have been achieved only in large referral centers with experience in hypothermia use and when treatment was applied within a few hours after an injury [66]. In ischemic stroke, animal studies and some clinical data suggest that hypothermia could limit neurological injury, but insufficient evidence exists to recommend its use outside the context of clinical trials. Several non-controlled small feasibility studies have used mild hypothermia in patients with ischemic stroke and 
all investigators reported significant decreases in brain edema and improved outcome compared with historical controls [67]. At our institution, a pilot study is underway to investigate the safety and feasibility of inducing and maintaining mild hypothermia in severe stroke patients (Rapid Acute Stroke Hypothermia Induction (RASHI) Trial). The POLAR trial which is a multi-center randomized trial currently recruiting patients has also been developed to investigate whether early cooling of patients with severe traumatic brain injury is associated with better outcomes. The Eurotherm3235 trial is another international, multicenter randomized controlled trial which will examine the effects of titrated therapeutic hypothermia $\left(32-35^{\circ} \mathrm{C}\right)$ as a treatment for raised intracranial pressure after traumatic brain injury.

\section{REMOVAL OF CSF}

If the CSF compartment is contributing to the elevated ICP, as in the case of obstructive or communicating hydrocephalus from SAH or intraventricular hemorrhage; the treatment strategy of choice is CSF diversion. This can be accomplished with an external ventricular drainage device (EVD), lumbar drain, or serial LP. Rapid aspiration of CSF should be avoided because it may lead to obstruction of the catheter opening by brain tissue. Also, in patients with aneurysmal subarachnoid hemorrhage, abrupt lowering of the pressure differential across the aneurysm dome can precipitate recurrent hemorrhage. A lumbar drain is generally contraindicated in the setting of high ICP due to the risk of transtentorial herniation.

\section{DECOMPRESSIVE CRANIECTOMY}

When managing a patient with increased ICP, an emergent compressive craniectomy may be considered if the patient is deteriorating rapidly or if the ICP continues to rise despite ongoing medical management. The decision to perform decompressive surgery should, however, be made on an individual basis in every patient. Studies suggest that some patients with expanding focal mass lesions and rapidly progressive herniation syndromes could benefit from emergent decompressive craniectomy and mass resection. Obvious mass lesions associated with an elevated ICP should be removed, if possible [5]. Different studies suggest that rapid and sustained control of ICP, including the use of decompressive craniectomy, improves outcomes in trauma, stroke, and subarachnoid hemorrhage in carefully selected cases $[68,69]$. Based on a meta-analysis of patients from three European randomized controlled trials; DECIMAL (DEcompressive Craniectomy In MALignant middle cerebral artery infarction), DESTINY (DEcompressive Surgery for the Treatment of malignant INfarction of the middle cerebral arterY), and HAMLET (the Hemicraniotoy After Middle Cerebral Artery infarction with Lifethreatening Edema Trial) surgical decompression reduces case fatality and poor outcome in patients with spaceoccupying infarctions. Individual data for patients aged between 18 and 60 years, with space-occupying MCA infarction, included in one of the three trials, and treated within $48 \mathrm{~h}$ after stroke onset were pooled for analysis. Included patients had clinical deficits suggestive of infarction in the territory of the MCA with a score on the National Institutes of Health Stroke Scale (NIHSS) $>15$, decrease in the level of consciousness to a score of 1 or greater on item 1a of the NIHSS, signs of an infarct on CT of at least $50 \%$ of the MCA territory, with or without additional infarction in the territory of the anterior or posterior cerebral artery on the same side, or infarct volume $>145 \mathrm{~cm}^{3}$ as shown on diffusion-weighted MRI. 93 patients were included in the pooled analysis. The effect of surgery was highly consistent across the three trials. In patients with malignant MCA infarction, decompressive surgery undertaken within $48 \mathrm{~h}$ of stroke onset reduces mortality and increases the number of patients with a favorable functional outcome [70, 71]. In DECRA trial, a randomized trial of adults with severe diffuse traumatic brain injury and refractory intracranial hypertension, early bifrontotemporoparietal decompressive craniectomy decreased intracranial pressure and the length of stay in the ICU but was associated with more unfavorable outcomes [71]. These conclusions were not really supported by closer examination of the basic data. Some authorities have claimed that DECRA trial results should have no influence on clinical practice. The ongoing RESCUEicp (Randomised Evaluation of Surgery with Craniectomy for Uncontrollable Elevation of IntraCranial Pressure) study hopes to address this issue [72].

\section{CONCLUSIONS}

Increased intracranial pressure is a neurologic emergency caused by a variety of neurologic injuries and is associated with worse outcomes, including brain ischemia and death. Prompt diagnosis, careful analysis of the underlying pathophysiology, and invasive monitoring and therapy of this condition are essential for successful management of this potentially devastating condition. Invasive methods of diagnosis and monitoring have their own risks. Recent technical innovations in non-invasive diagnosis and assessment of raised ICP may allow for improvement in morbidity and mortality rates, but need to be examined in large clinical trials before become the standard of care. To date there are few interventions which showed efficacy in reducing ICP but not all have proven to improve outcomes. The risks and benefits of medical and surgical interventions must be carefully evaluated and the best treatment options must be tailored for each patient.

\section{CONFLICT OF INTEREST}

The authors confirm that this article content has no conflict of interest.

\section{ACKNOWLEDGEMENTS}

Declared none.

\section{REFERENCES}

[1] Treggiari MM, Schutz N, Yanez ND, Romand J-A. Role of intracranial pressure values and patterns in predicting outcome of traumatic brain injury: a systematic review. Neurocrit Care 2007; 6: 104-12.

[2] Ropper AH. Hyperosmolar Therapy for Raised Intracranial Pressure. N Engl J Med 2012; 367: 746-52.

[3] Bratton SL, Chestnut RM, Ghajar J, et al. Intracranial pressure thresholds. J Neurotrauma 2007; 24(Suppl 1): 55-8.

[4] Mayer SA, Chong JY. Critical care management of increased intracranial pressure. J Intensive Care Med 2002; 17: 55-67.

[5] Nakagawa K, Smith WS. Evaluation and management of increased intracranial pressure. Continuum Lifelong Learning Neurol 2011; 17(5): 1077-93. 
[6] Ropper AH. Hyperosmolar Therapy for raised intracranial pressure. N Engl J Med 2012; 367: 746-52.

[7] Franco FA. Cerebral autoregulation and syncope. Prog Cardiovasc Dis 2007; 50: 49-80.

[8] Lassen NA, Agnoli A. The upper limit of autoregulation of cerebral blood flow--on the pathogenesis of hypertensive encepholopathy. Scand J Clin Lab Invest 1972; 30: 113.

[9] Eisenberg HM, Gary HE Jr, Aldrich EF, et al. Initial CT findings in 753 patients with severe head injury. A report from the NIH Traumatic Coma Data Bank. J Neurosurg 1990; 73: 688.

[10] Narayan RK, Kishore PR, Becker DP, et al. Intracranial pressure: To monitor or not to monitor? A review of our experience with severe head injury. J Neurosurg 1982; 56: 650-59

[11] Chesnut RM, Temkin N, Carney N, et al. A trial of intracranialpressure monitoring in traumatic brain injury. N Engl J Med 2012; 367: 2471-81.

[12] Bratton SL, Chestnut RM, Ghajar J, et al. Guidelines for the management of severe traumatic brain injury. VII. Intracranial pressure monitoring technology. J Neurotrauma 2007; 24(Suppl 1): S45.

[13] Mayhall CG, Archer NH, Lamb VA, et al. Ventriculostomy related infections: a prospective epidemiologic study. N Engl J Med 1984; 310: 553-9.

[14] Ostrup RC, Luerssen TG, Marshall LF, Zornow MH. Continuous monitoring of intracranial pressure with a miniaturized fiberoptic device. J Neurosurg 1987; 67: 206.

[15] Gambardella G, d'Avella D, Tomasello F. Monitoringof brain tissue pressure with a fiberopticdevice. Neurosurgery 1992; 31: 918.

[16] Bochicchio M, Latronico N, Zappa S, et al. Bedside burr hole for intracranial pressure monitoring performed by intensive care physicians. A 5-year experience. Intensive Care Med 1996; 22: 1070

[17] Ream AK, Silverberg GD, Corbin SD, et al. Epidural measurement of intracranial pressure. Neurosurgery $1979 ; 5: 36-43$.

[18] Blei AT, Olafsson S, Webster S, Levy R. Complications of intracranial pressure monitoring in fulminant hepatic failure. Lancet 1993; 341: 157.

[19] Wolfe TJ, Torbey MT. Mnagement of Intracranial Pressure, Curr Neuro Neurosci Reports 2009; 9: 477-85.

[20] Hayashi M, Handa Y, Kobayashi H, et al. Plateau-wave phenomenon (I). Correlation between the appearance of plateau waves and CSF circulation in patients with intracranial hypertension. Brain 1991; 114(Pt 6): 2681.

[21] Rosner MJ, Becker DP. Origin and evolution of plateau waves. Experimental observations and a theoretical model. J Neurosurg 1984; 60: 312.

[22] Rosenberg JB, Shiloh AL, Savel RH, Eisen LA. Non-invasive metods of estimating intracranial pressure. Neurocrit care 2011; 15(3): 599-608.

[23] Hassler W, Steinmetz H, Gawlowski J. Transcranial Doppler ultrasonography in raised intracranial pressure and in intracranial circulatory arrest. J Neurosurg 1988; 68: 745-51.

[24] Dubost C, Motuel J, Geeraerts T. Non invasive evaluation of intracranial pressure: How and for whom? Ann Fr Anesth Reanim 2012; 31(6): e125-32.

[25] Al-Jehani HM, Marcoux J, Angle MR, Teitelbaum JS. The use of transcranial Doppler pulsatility index to guide hyperosmolar therapy. Neurosciene (Riyadh) 2012; 17: 363-7.

[26] Manno EM. Transcranial doppler ultrasonography in the neurocritical care unit. Crit Care Clin 1997; 13: 79.

[27] Edouard AR, Vanhille E, Le Moigno S, et al. Non-invasive assessment of cerebral perfusion pressure in brain injured patients with moderate intracranial hypertension. Br J Anaesth 2005; 94: 216.

[28] Mayer SA, Thomas CE, Diamond BE. Asymmetry of intracranial hemodynamics as an indicator of mass effect in acute intracerebral hemorrhage: a transcranial Doppler study. Stroke 1996; 27: 178892.

[29] Bellner J, Romner B, Reinstrup P, Kristiansson KA, Ryding E, Brandt L. Transcranial doppler sonography pulsatility index (pi) reflects intracranial pressure (ICP). Surg Neurol 2004; 62: 45-51.

[30] Martí-Fàbregas J, Belvís R, Guardia E, Cocho D, Muñoz J, Marruecos L, Martí-Vilalta JL. Prognostic value of Pulsatility Index in acute intracerebral hemorrhage. Neurology 2003; 61: 1051-6.
[31] Schmidt B, KlingelhoÈfer J, Schwarze JJ, et al. Noninvasive prediction of intracranial pressure curves using transcranial doppler ultrasonography and blood pressure curves. Stroke 1997; 28: 2465 72.

[32] Sloan MA, Alexandrov AV, Tegeler CH, et al. Therapeutics and Technology assessment subcommittee of the American academy of neurology. Assessment: transcranial doppler ultrasonography: report of the therapeutics and technology assessment subcommittee of the American academy of neurology. Neurology 2004; 62(9): 1468-81.

[33] Loch MR. Management of cerebral vasospasm. Neurosurg Rev 2006; 29(3); 179-93.

[34] Rajajee V, Vanaman M, Fletcher JJ, Jacobs TL. Optic nerve ultrasound for the detection of raised intracranial pressure. Neurocrit Care 2011; 15(3): 506-15.

[35] Dubourg J, Javouhey E, Geeraerts T, et al. Ultrasonography of optic nerve sheath diameter for detection of raised intracranial pressure: a systematic review and meta-analysis. Intens Care Med 2011; 37: 1059 .

[36] Kiphuth IC, Huttner HB, Breuer L, Schwab S, Köhrmann M. Sonographic monitoring of midline shift predicts outcome after intracerebral hemorrhage. Cerebrovasc Dis 2012; 34: 297-304.

[37] Friedman D, Claassen J, Hirsch LJ. Continuous electroencephalogram monitoring in the intensive care unit. Anesth Analg; 2009; 109: 506-23.

[38] Amantini A, Fossi S, Grippo A, etal. Continuous EEG-SEP monitoring in severe brain injury. Neurophysiol Clin 2009: 39: 8593.

[39] Roberts I, Schierhout G, Alderson P. Absence of evidence for the effectiveness of five interventions routinely used in the intensive care management of severe head injury: a systematic review. J Neurol Neurosurg Psychiat 1998; 65(5): 729-33.

[40] Ker K, Perel P, Blackhall K, et al. How effective are some common treatments for traumatic brain injury? BMJ 2008; 337: a865

[41] Bratton SL, Chestnut RM, Ghajar J, et al.: Cerebral perfusion thresholds. J Neurotrauma 2007; 24(Suppl 1): 59-64.

[42] Moraine J, Berre J, Melot C. Is cerebral perfusion pressure a major determinant of cerebral blood flow during head elevation in comatose patients with severe intracranial lesions? J Neurosurg 2000; 92: 606-614.

[43] Rosner MJ, Rosner SD, Johnson AH. Cerebral perfusion pressure: management protocol and clinical results. J Neurosurg 1995; 83: 949-62.

[44] SAFE Study Investigators, Australian and New Zealand Intensive Care Society Clinical Trials Group, Australian Red Cross Blood Service, et al. Saline or albumin for fluid resuscitation in patients with traumatic brain injury. N Engl J Med 2007; 357: 874.

[45] Lassen NA, Christensen MS. Physiology of cerebral blood flow. Br J Anaesth 1976; 48: 719.

[46] Busija DW, Leffler CW, Pourcyrous M. Hyperthermia increases cerebral metabolic rate and blood flow in neonatal pigs. Am J Physiol 1988; 255: H343.

[47] Busto R, Dietrich WD, Globus MY, et al. Small differences in intraischemic brain temperature critically determine the extent of ischemic injury. J Cereb Blood Flow Metab 1987; 7: 129-38.

[48] Schortgen F, Clabault K, Katsahian S, et al. Fever control using external cooling in septic shock: a randomized controlled trial. Am J RespirCrit Care Med 2012; 185: 1088-95.

[49] Claassen J, Mayer SA, Kowalski RG, Emerson RG, Hirsch LJ. Detection of electrographic seizures with continuous EEG monitoring in critically ill patients. Neurology 2004; 62(10): 1743 8

[50] Vespa PM, Miller C, McArthur D, et al. Nonconvulsive electrographic seizures after traumatic brain injury result in a delayed, prolonged increase in intracranial pressure and metabolic crisis. Crit Care Med 2007; 35: 2030-6

[51] Edwards P, Arango M, Balica L, et al. Final results of MRC CRASH, a randomised placebo-controlled trial of intravenous corticosteroid in adults with head injury-outcomes at 6 months. Lancet 2005; 365(9475): 1957-9.

[52] Honda H, Warren DK. Central nervous system infections: meningitis and brain abscess. Infect Dis Clin North Am. 2009 Sep; 23(3): 609-23

[53] Nath F, Galbraith S. The effect of mannitol on cerebral white matter water content. J Neurosurg 1986; 65(1): 41Y43. 
[54] Paczynski RP. Osmotherapy; basic concepts and controversies. Crit Care Clin 1997; 13(1): 105Y129.

[55] Torre-Healy A, Marko NF, Weil RJ. Hyperosmolar therapy for intracranial hypertension. Neurocrit Care 2012; 17: 117-30.

[56] Oddo M, Levine JM, Frangos S, et al. Effect of mannitol and hypertonic saline on cerebral oxygenation in patients with severe traumatic brain injury and refractory intracranial hypertension. $\mathrm{J}$ Neurol Neurosurg Psychiatry 2009; 80: 916-20.

[57] Kamel H, Navi BB, Nakagawa K, et al. Hypertonic saline versus mannitol for the treatment of elevated intracranial pressure: a metaanalysis of randomized clinical trials. Crit Care Med 2011; 39: 554.

[58] García-Morales EJ, Cariappa R, Parvin CA, et al. Osmole gap in neurologic-neurosurgical intensive care unit: its normal value, calculation, and relationship with mannitol serum concentrations. Crit Care Med 2004; 32: 986-91.

[59] Latorre JG, Greer DM. Management of acute intracranial hypertension: a review. Neurologist 2009; 15(4): 193 Y207.

[60] Smith R, Thomas M, Brown J: Raised intra-cranial pressuresodium bicarbonate as an alternative hyperosmolar treatment. J Neurosurg Anesthesiol 2008, 20: 158.

[61] Stocchetti N, Maas AI, Chieregato A, van derPlas AA. Hyperventilation in head injury: a review. Chest 2005; 127: 1812.

[62] Muizelaar JP, Marmarou A, Ward JD, et al. Adverse effects of prolonged hyperventilation in patients with severe head injury: a randomized clinical trial. J Neurosurg 1991; 75: 731

[63] Muizelaar JP, van der Poel HG, Li ZC, et al. Pial arteriolar vessel diameter and $\mathrm{CO} 2$ reactivity during prolonged hyperventilation in the rabbit. J Neurosurg 1988; 69: 923-7.

[64] Bratton SL, Chestnut RM, Ghajar J, et al. Guidelines for the management of severe traumatic brain injury. XI. Anesthetics, analgesics, and sedatives. J Neurotrauma 2007; 24(Suppl 1): S71S76.

[65] Eisenberg HM, Frankowski RF, Contant CF, et al. High-dose barbiturate control of elevated intracranial pressure in patients with severe head injury. J Neurosurg 1988; 69: 15.

[66] McIntyre LA, Fergusson DA, Hebert PC, et al. Prolonged therapeutic hypothermia after traumatic brain injury in adults: a systematic review. JAMA 2003; 289: 2992-9.

[67] Polderman KH. Induced hypothermia and fever control for prevention and treatment of neurological injuries. Lancet 2008; 371: 1955-69.

[68] Hofmeijer J, Kappelle LJ, Algra A, et al. Surgical decompression for space-occupying cerebral infarction (the Hemicraniectomy After Middle Cerebral Artery infarction with Life-threatening Edema Trial [HAMLET]): a multicentre, open, randomised trial. Lancet Neurol 2009; 8: 303-4.

[69] Huang AP, Tu YK, Tsai YH, et al. Decompressive craniectomy as the primary surgical intervention for hemorrhagic contusion. J Neurotrauma 2008; 25: 1347-54

[70] Vahedi K, Hofmeijer J, Juettler E, et al. Early decompressive surgery in malignant infarction of the middle cerebral artery: a pooled analysis of three randomised controlled trials. Lancet Neurol 2007; 6(3): 215-22

[71] Cooper DJ, Roseneld JV, Murray L, et al. Decompressive Craniectomy in Diffuse Traumatic Brain Injury. N Engl J Med 2011; 364: 1493-1502.

[72] Honeybul S, Ho KM, Lind CR. What can be learned from the DECRA study. World Neurosurg 2013; 79(1): 159-61.

This is an open access article licensed under the terms of the Creative Commons Attribution Non-Commercial License (http://creativecommons.org/licenses/by-nc/ 3.0/) which permits unrestricted, non-commercial use, distribution and reproduction in any medium, provided the work is properly cited. 